\title{
Análise Empírica de Tendências na Produção Científica sobre Coping (SciELO, 1993/2012)
}

\author{
Fabíola Ribeiro de Moraes Santeiro ${ }^{1}$ \\ Daniela Sacramento Zanini \\ Pontifícia Universidade Católica de Goiás \\ Tales Vilela Santeiro \\ Universidade Federal do Triângulo Mineiro
}

\begin{abstract}
RESUMO - Coping indica uma ação intencional, física ou mental, iniciada em resposta a um estressor percebido, dirigida para circunstâncias externas ou estados internos. A pesquisa visou a obter panorama sistemático do que tem sido estudado e discutido em parte da produção científica sobre coping, mediante a análise de artigos publicados em periódicos indexados à base de dados SciELO, entre 1993 e 2012 ( $N=294)$. Observou-se aumento quantitativo das produções no período investigado e elas foram divulgadas predominantemente em periódicos da Psicologia. Autorias femininas e grupais, desenvolvendo estudos empíricos, foram expressivas. Transtornos orgânicos, instituições hospitalares e usuários de serviços de saúde foram mais investigados e discutidos. A terminologia coping nem sempre é enfocada como um corpo teórico específico, o que requer atenção do leitor.
\end{abstract}

Palavras-chave: estilos de enfrentamento, bases de dados, periódicos científicos, metaciência

\section{Empirical Analysis of Trends in Scientific Production on Coping (SciELO, 1993/2012)}

\begin{abstract}
Coping indicates an intentional action, physical or mental, initiated in response to a perceived stressor, addressed to external circumstances or internal states. The research aimed to obtain systematic survey of what has been studied and discussed in the scientific production about coping, through the analysis of articles published in journals indexed to the Database SciELO, between 1993 and 2012 ( $N=294)$. It was observed a quantitative increasing of the productions in the investigated period and they were predominantly reported in journals of psychology. Female and groupal authorship, developing empirical studies were significant. Organic disorders, hospitals and health service users were more investigated and discussed. The terminology is not always focused coping as a specific theoretical framework, which requires attention of the reader.
\end{abstract}

Keywords: coping strategies, databases, journals, metascience

Vive-se na atualidade em um mundo globalizado, no qual as informações, independentemente de sua origem, circulam com grande facilidade. A um cidadão comum, por exemplo, é possível acessar via internet um conteúdo qualquer que lhe aprouver, o que, contudo, não garantirá a qualidade científica da informação. Neste sentido, espera-se que a academia, de onde se aspira que os conhecimentos gerados primem pela qualidade, cumpra seu papel na promoção e divulgação do saber, cuidando para que este promova desenvolvimento.

O progresso científico vincula-se, assim, à divulgação de descobertas e invenções. E para que isso ocorra, novos fatos devem ser disponibilizados à comunidade acadêmica. Com esses propósitos, é necessário reunir e divulgar as informações para aqueles que estudam determinado assunto (Sabadini, Sampaio, \& Koller, 2009). Nesse cenário a divulgação científica tem a função de trazer e elucidar fatos científicos aos próprios cientistas ou aos leigos, para que possam se atualizar em suas áreas ou em outras áreas do conhecimento. Essa possibilidade tem sido facilitada por iniciativas como as bibliotecas de acesso aberto e disponibilizadas por acesso eletrônico em rede (Packer, 2009, 2011).

1 Endereço para correspondência: Rua 9, n.769, Setor Oeste, Goiânia, Goiás, Brasil. CEP: 74110-100.E-mail: dazanini@yahoo.com
O pesquisador ao escolher seu objeto de estudo pode fazer essa seleção a partir de vários motivos, dentre eles, a afinidade pessoal, a conveniência, os modismos temáticos etc. Entretanto, quando se pensa na evolução do conhecimento, ela efetiva-se de fato a partir de alguns critérios, e a produção científica é um deles. Esta última tem sido entendida como aquelas atividades que resultam em novas descobertas tecnológicas e/ou de conhecimentos, assim como no aumento das informações disponíveis para direcionar os progressos do homem (Poblacion, Witter, \& Silva, 2006).

A análise de produção científica é, nesse sentido, uma metodologia que permite sinalizar como e quanto tem sido pesquisado e estudado em determinada área do conhecimento. Ela permite averiguar, por conseguinte, os rumos mais adequados às novas pesquisas necessárias para o crescimento deste setor em estudo.

O início da década de 1960 tem sido apontado como o momento em que a avaliação e análise da produção científica que utiliza metodologia específica surgiram (Domingos, 1999). Desde então esse tipo de pesquisa vem evoluindo consideravelmente, possibilitando tanto análises qualitativas, quanto quantitativas, em várias áreas do conhecimento, assim como em instituições produtoras de ciência. O estudo realizado por Granja (1995) sugeria, àquela época, que o interesse por pesquisas sobre produção científica, realizadas 
em universidades brasileiras, era constatado com maior ênfase nos últimos cinco anos. No ínterim entre o estudo de Granja e os dias atuais, muito se modificou nesse sentido, o que pode ser visualizado na intensificação de produções com esse enfoque, por pesquisadores de diversas instituições brasileiras (Barroso, 2010; Custódio, Crepaldi, \& Cruz, 2012; Joly, Berberian, Andrade, \& Teixeira, 2010). Por intermédio de análises de produção pode-se avaliar o estágio de desenvolvimento de um país e de instituições (Herculano \& Norberto, 2012), de periódicos (Pinto, de Lima, \& de Lima, 2011), de um dado pesquisador (Massi \& Queiroz, 2012) e de temáticas específicas (Aros \& Yoshida, 2009), assim como, neste momento se propõe analisar textos sobre coping.

\section{Coping}

Coping pode ser definido como o conjunto de esforços, cognitivos e comportamentais, utilizados pelo indivíduo visando a lidar com demandas específicas, internas ou externas, que surgem em situações de estresse e são avaliadas como sobrecarga ou excedente aos recursos pessoais (Lazarus \& Folkman, 1984). Esta definição proposta por Lazarus e Folkman (1984), e amplamente reproduzida nas publicações sobre o tema, enfatiza o caráter processual do coping e destaca quatro aspectos fundamentais da teoria: (a) processo ou interação do indivíduo em relação ao seu ambiente; (b) função: lidar com a situação estressora, ao invés de controlá-la ou dominá-la; (c) processo pressupõe avaliação: como o fenômeno é percebido, interpretado e cognitivamente representado; e (d) processo de mobilização de esforços cognitivos e comportamentais para administrar (reduzir, minimizar ou tolerar) demandas internas ou externas surgidas da interação com o ambiente.

Assim, pode-se dizer que coping é uma ação intencional, física ou mental, iniciada em resposta a um estressor percebido, dirigida para circunstâncias externas ou estados internos (Lazarus \& Folkman, 1984). Neste sentido, as estratégias de coping refletem ações, comportamentos ou pensamentos usados para lidar com um estressor.

O processo de coping é desencadeado apenas se o problema a ser enfrentado é considerado um estressor. Caso contrário, ou seja, caso o evento não seja apreciado como um problema ou um evento estressante (ou estressor), o que se tem é um processo de adaptação (Lazarus \& Folkman, 1984). Contudo, vale lembrar que coping é considerado qualquer tentativa de administrar o evento estressor, tenha ela sucesso ou não. Assim, uma característica dos processos de coping não é o resultado, mas sim a apreciação realizada do problema (se estressor ou não).

A escolha da estratégia de coping depende da análise da situação na qual o indivíduo se envolve. Esta análise é chamada na literatura da área de avaliação ou appraisal e é classificada como avaliação primária e secundária. $\mathrm{Na}$ avaliação primária o indivíduo aprecia se o problema enfrentado constitui-se em um evento estressante (Lazarus \& Folkman, 1984).

A disponibilidade de recursos de coping afeta a avaliação do evento (se estressante ou não) e determina que estratégias de coping possam ser utilizadas. Neste caso, ocorre a avaliação secundária. Nela o indivíduo avalia os recursos de coping existentes e seleciona a melhor estratégia para fazer frente ao problema. Os processos de coping variam com o desenvolvimento: condições e experiências de vida, fases do ciclo vital etc.

Em seus estudos Lazarus e Folkman (1984) postularam dois conjuntos de estratégias de coping que poderiam ser definidas segundo sua função: (a) focado na emoção: esforço para regular o estado emocional que é associado ao estresse. Nesse tipo de estratégia de coping objetiva-se alterar o estado emocional relacionado ao problema enfrentado pelo indivíduo. Alguns autores também descrevem esse conjunto de estratégias como de evitação do problema (Moos, 1993; 2002; 2003). E (b) focado no problema: esforço para atuar na situação que originou o estresse, tentando modificá-la, principalmente administrando, lidando com a situação, pois algumas não podem ser mudadas. Esse conjunto de estratégias de coping visa a alterar o problema existente na relação entre a pessoa e o ambiente causador da tensão. Esse conjunto de estratégias também é referida em alguns trabalhos como de aproximação do problema ou de enfrentamento direto (Moos, 1993, 2002, 2003).

Considerando a interface possível entre produção científica e necessidades de avaliá-la na área de coping, bem como a inexistência de estudos com esse enfoque na realidade brasileira, este estudo objetivou, de modo geral, delinear sistematicamente o panorama da produção científica sobre coping, mediante a análise de artigos publicados em periódicos indexados à base de dados eletrônica Scientific Electronic Library Online (Biblioteca Científica Eletrônica em Linha [SciELO], 2012), de agora em diante SciELO.

De modo mais específico, as seguintes indagações nortearam o processo de investigação: (a) Como a produção sobre coping se distribui, por ano? Entendendo que a SciELO representa parcela relevante de produções nacionais e estrangeiras, pode ser dito que estudos sobre coping têm aumentado? (b) Quais periódicos têm divulgado estudos sobre coping? Estudos sobre coping têm sido veiculados em periódicos de Psicologia, ou também o fazem naqueles de responsabilidade editorial de outras áreas? (c) Os autores têm pesquisado em grupos ou isoladamente? Como eles se caracterizam quanto ao sexo? (d) Que tipo de estudos sobre coping têm sido desenvolvidos e publicados? Essa área temática tem se consolidado por meio de pesquisas empíricas? (e) Produções que versam sobre coping são, de fato, específicas sobre coping, ou esse vocábulo tem sido adotado de modo inespecífico, pelos autores? E (f) Quais temáticas, locais e segmentos populacionais têm sido estudados?

\section{Método}

\section{Material}

A amostra do estudo é composta por 294 artigos sobre coping, publicados nos últimos 20 anos (1993-2012), em 49 periódicos indexados à SciELO. 


\section{Critérios de Inclusão e de Exclusão}

Quaisquer produções sobre coping foram incluídas na amostra, publicadas em qualquer idioma, desde que: (a) acessíveis por meio das palavras-chave coping ou enfrentamento ou enfrentamiento, no campo "todos os índices" do "formulário básico"; e (b) estivessem disponíveis online até março de 2012, quando a busca de informações foi encerrada. Exceto por essa necessidade de estabelecer o prazo para fechar o tamanho da amostra e prosseguir nas demais etapas da pesquisa, não houve demais restrições de período temporal para realização das buscas. Essa decisão foi tomada para aperfeiçoar as probabilidades de obtenção de um panorama abrangente do que tem sido produzido e discutido sobre coping, com indexação na SciELO. Nesse sentido, 1993 correspondeu ao ano que acusou o primeiro resultado.

Os critérios de exclusão de material foram aplicados a duas situações. Na primeira delas os artigos veiculavam informação em periódicos de áreas do conhecimento distintas da saúde e da Psicologia. Assim, produções constantes dos seguintes periódicos foram excluídas: Perspectivas em Ciência da Informação; Brazilian Journal of Biology; Scientia Agricola; Journal of Information Systems and Technology Management; Revista Brasileira de Botânica; Perspectivas em Ciência da Informação; Brazilian Adminstration Review; Revista de Administração Mackenzie; Revista de Administração de Empresas; e Revista Brasileira de Educação.

Na segunda situação de exclusão, mesmo as produções tendo sido publicadas em periódicos da área da saúde e da Psicologia, o teor das informações não mantinha relação com alguma teoria ou ideia sobre coping. A relação de periódicos excluídos foi: Brazilian Oral Research; Brazilian Dental Journal; Brazilian Journal of Medical and Biological Research; Journal of Applied Oral Science; Jornal Brasileiro de Pneumologia; e Revista CEFAC: Atualização Científica em Fonoaudiologia e Educação.

Atentando-se a essas particularidades, em síntese, 17 revistas foram excluídas da amostra de periódicos, inicialmente composta por 68 títulos. Por conseguinte, nessas revistas descartadas, 19 artigos foram desconsiderados, porque $11(64,7 \%)$ deles se enquadravam no critério 1 de exclusão e seis $(35,3 \%)$ no critério 2 .

\section{Análise por Juízes}

Para verificar o grau de fidedignidade do julgamento do item referente a quanto o uso da terminologia sobre o construto coping era específico (alinhado às teorias propostas por Folkman \& Lazarus, 1980; Lazarus \& Folkman, 1984; Moos, 2002), dois juízes realizaram avaliações independentes: a primeira autora e um pesquisador experiente na metodologia de análise de produção científica (terceiro autor). Para análise dos demais itens, os quais consistiam em contagem simples de frequências e facilmente revisáveis, recorreu-se à avaliação pelas duas primeiras autoras.

\section{Base de Dados SciELO}

A SciELO é um modelo de publicação eletrônica para países em desenvolvimento, que se pauta na crença de que o acesso adequado e atualizado à informação técnicocientífica é essencial para o desenvolvimento econômico e social. Ela foi especialmente desenvolvida para responder às necessidades da comunicação científica; visa à publicação eletrônica cooperativa de periódicos científicos na internet e proporciona soluções para assegurar a visibilidade e o acesso universal à literatura científica gerada nos países em desenvolvimento e particularmente na América Latina e Caribe (SciELO, 2012).

Historicamente, a SciELO surgiu de um projeto piloto que envolvia 10 periódicos brasileiros de diferentes áreas do conhecimento (Packer et al., 1998; Packer, 2009). Ela tem sido desenvolvida com êxito entre março de 1997 e maio de 1998 e tem se aprimorado até os dias atuais. Nessa direção, salienta-se que as coleções de periódicos SciELO indexam e publicam as melhores revistas da maioria dos países produtores de pesquisa da América Latina e do Caribe, além de Portugal e Espanha. Em 2009, a África do Sul juntou-se à rede (Packer, 2009).

Vale observar que os periódicos das coleções SciELO ainda seguem padrões editoriais de nível internacional, não somente avaliados pelos comitês consultivos da própria SciELO. Em alguns casos há sobreposição de avaliações, por exemplo, por aquelas realizadas dentro do modelo Qualis/ CAPES (Coordenação de Aperfeiçoamento de Pessoal de Nível Superior, 2013).

\section{Procedimentos}

Os procedimentos compreenderam cinco etapas: (1) levantamento e acesso às produções, por meio do vocábulo/descritor coping; (2) leitura prévia do material coletado e eliminação de duplicidades e impertinências; (3) categorização dos achados, considerando-se os objetivos propostos; (4) verificação da significância estatística das ocorrências, por meio do teste estatístico não-paramétrico Qui-quadrado (Siegel, 1956/1975), momento em que o $\mathrm{p}$ foi preestabelecido em 0,05 ; e (5) discussão quanti-qualitativa dos achados.

Sobre o refinamento do material conforme a especificidade de análise/estudo sobre coping, a quantidade de artigos avaliada pelos juízes foi de aproximadamente $15 \%$ do total da amostra (45 artigos), obtidos por sorteio, sem reposição (um determinado artigo sorteado não era reintegrado à amostra dos 45 artigos, para evitar que fosse reavaliado). Antes de a avaliação efetiva ocorrer, treinamentos foram realizados com base em outros 15 artigos, sorteados de modo semelhante àqueles anteriormente descritos.

Após o treino e a obtenção de concordância ter sido refinada é que se realizaram os julgamentos definitivos, com base no índice Kappa (Bordens \& Abbott, 1999). Assim, a primeira autora procedeu à análise do restante do material (249 artigos) somente após o índice de concordância entre as avaliações feitas pelo par de juízes ter sido obtido. 
Considerada essa particularidade, o Kappa obtido entre os juízes, em seus julgamentos independentes, foi de 0,91 , o que sinalizou que as avaliações realizadas desfrutavam de fidedignidade.

\section{Resultados e Discussão}

\section{Distribuição da Produção sobre Coping}

A produção analisada teve início no ano de 1993, momento no qual um estudo foi identificado, o que se repetiu em 1994. Em 1995 e em 1997 não foram observados estudos sobre coping. Considerados esses primeiros resultados, depois de 1996 a produção analisada foi, no geral, crescente, em especial a partir dos anos 2000, ano em que quatro produções foram constatadas. Nesse sentido, o ano de 2011 indicou o número máximo de artigos produzidos sobre coping. Como ressaltado anteriormente, a busca de informações foi finalizada em março de 2012, de modo que esse resultado precisa ser assim considerado. Isso posto, a média anual de publicações sobre coping foi de 16,3 artigos ( $\mathrm{DP}=15,96)$.

Quando se compara a produção observada nas diferentes décadas (Tabela 1), verifica-se que 3,7\% situam-se nos oito anos compreendidos entre 1993 e 2000 (média de dois trabalhos/ano; $D P=1,21$ ), enquanto na década seguinte 23 $(72,8 \%)$ trabalhos/ano foram constatados $(D P=11,81)$. Nos anos restantes a média saltou para $26(18,1 \%)$ trabalhos/ano.

O incremento significativo da produção dos anos 2000 em diante é entendido de duas formas básicas: (a) a contínua expansão da SciELO, com a indexação de novos periódicos desde sua criação (Packer, 2009; SciELO, 2012); e (b) a também sucessiva aceitação e adaptação do modelo teórico proposto por pesquisadores de coping. Com isso, não se pode considerar que houve propriamente apenas um aumento de interesse pelo tema coping no período relatado, mas que a visibilidade da produção existente se intensificou. Esse tipo de constatação também ocorre em outros estudos que tomam bases de dados eletrônicas como fonte material de pesquisas (por exemplo, Yoshida, Santeiro, Santeiro, \& Rocha, 2005), pois não pode ser desvinculada do movimento representado por iniciativas de acesso aberto a publicações científicas, que tem sido consolidado no meio acadêmico brasileiro de modo progressivo, não somente por modelos como o da SciELO, mas por outros semelhantes a ele (Sampaio \& Serradas, 2009), entre os quais destaca-se a base de dados PePsic, específica da área de Psicologia.

\section{Periódicos Divulgadores}

No período investigado, o periódico que mais publicou sobre coping foi a Revista Latino-Americana de Enfermagem: 38 artigos (12,9\%). Em outro extremo, 18 veículos apresentaram frequência de um artigo publicado ( $0,3 \%$ cada), conforme Tabela 2. Isso resulta em uma média de seis artigos/ periódico $(D P=7,62)$ no período estudado.

A produção sobre coping está concentrada em sete periódicos (51,4\%): a já citada Revista Latino-Americana de
Tabela 1. Distribuição da produção sobre coping, por ano

\begin{tabular}{|c|c|c|c|c|}
\hline $\begin{array}{c}\text { Ano da } \\
\text { Produção }\end{array}$ & $\mathbf{F}$ & $\%$ & $\begin{array}{c}\text { F } \\
\text { Acumulada }\end{array}$ & $\begin{array}{c}\% \\
\text { Acumulada }\end{array}$ \\
\hline 1993 & 1 & 0,3 & 1 & 0,3 \\
\hline 1994 & 1 & 0,3 & 2 & 0,6 \\
\hline 1995 & -- & -- & -- & -- \\
\hline 1996 & 2 & 0,7 & 4 & 1,3 \\
\hline 1997 & -- & -- & -- & -- \\
\hline 1998 & 1 & 0,3 & 5 & 1,6 \\
\hline 1999 & 2 & 0,7 & 7 & 2,3 \\
\hline 2000 & 4 & 1,4 & 11 & 3,7 \\
\hline $\begin{array}{c}\text { Subtotal } \\
\text { Anos } 1990\end{array}$ & 11 & 3,7 & 11 & 3,7 \\
\hline 2001 & 12 & 4,1 & 23 & 7,8 \\
\hline 2002 & 7 & 2,4 & 30 & 10,2 \\
\hline 2003 & 12 & 4,1 & 42 & 14,3 \\
\hline 2004 & 14 & 4,8 & 56 & 19,1 \\
\hline 2005 & 20 & 6,8 & 76 & 25,9 \\
\hline 2006 & 18 & 6,1 & 94 & 32,0 \\
\hline 2007 & 34 & 11,6 & 128 & 43,6 \\
\hline 2008 & 36 & 12,2 & 164 & 55,8 \\
\hline 2009 & 36 & 12,2 & 200 & 68,0 \\
\hline 2010 & 41 & 14,0 & 241 & 82,0 \\
\hline $\begin{array}{c}\text { Subtotal } \\
\text { Anos } 2000\end{array}$ & 230 & 78,2 & 241 & 82,0 \\
\hline 2011 & 52 & 17,7 & 293 & 99,7 \\
\hline 2012 & 1 & 0,3 & 294 & 100 \\
\hline $\begin{array}{c}\text { Subtotal } \\
\text { Anos } 2010\end{array}$ & 53 & 18,1 & 294 & 100 \\
\hline Total Geral & 294 & 100 & 294 & 100 \\
\hline
\end{tabular}

Enfermagem e outros seis - Psicologia: Reflexão e Crítica, Revista da Escola de Enfermagem da USP; Estudos de Psicologia de Campinas; Psicologia: Teoria e Pesquisa; Psicologia em Estudo; e Estudos de Psicologia de Natal. Observa-se que todos os veículos publicadores, não somente os sete acima, além de atenderem aos padrões editoriais da SciELO, são avaliados pela CAPES, num processo avaliativo denominado Qualis, conferindo a esses sete periódicos qualidade simbolizada por avaliações que vão de B1 a A1 (Tabela 2). Por essa via, deve ser observado que um mesmo periódico, ao ser classificado em duas ou mais áreas distintas, pode receber diferentes avaliações. Por exemplo, a Acta Paulista de Enfermagem é avaliada como A2 pela Enfermagem e como B1 pela Psicologia. Neste estudo considerou-se a avaliação Qualis/CAPES da área de Psicologia (Tabela 2).

Ainda sobre os veículos divulgadores, títulos de responsabilidade editorial da área de Medicina foram mais frequentes $(n=16 ; 32,7 \%)$, seguidos por periódicos da Psicologia $(n=11 ; 22,4 \%)$. Houve ainda, editores de Enfermagem ( $n=7 ; 14,3 \%)$, Educação $(n=6 ; 12,2 \%)$, Saúde Pública/Coletiva $(n=5 ; 10,2 \%)$ e Interdisciplinares $(n=4$; $8,2 \%$ ). Em todos os casos, os periódicos são sediados em instituições brasileiras. 
Tabela 2. Periódicos, em frequência decrescente de publicação de artigos, com respectiva avaliação Qualis/CAPES - área Psicologia (2013)

\begin{tabular}{|c|c|c|c|c|c|}
\hline Periódico & $\mathrm{F}$ & $\%$ & $\begin{array}{c}\mathrm{F} \\
\text { Acumulada }\end{array}$ & $\begin{array}{c}\% \\
\text { Acumulada }\end{array}$ & $\begin{array}{l}\text { Qualis/ } \\
\text { CAPES }\end{array}$ \\
\hline Revista Latino-Americana de Enfermagem & 38 & 12,9 & 38 & 12,9 & B1 \\
\hline Psicologia: Reflexão e Crítica & 24 & 8,2 & 62 & 21,1 & $\mathrm{~A} 1$ \\
\hline Revista da Escola de Enfermagem da USP & 24 & 8,2 & 86 & 29,3 & B1 \\
\hline Estudos de Psicologia de Campinas & 18 & 6,1 & 104 & 35,4 & $\mathrm{~A} 2$ \\
\hline Psicologia: Teoria e Pesquisa & 17 & 5,8 & 121 & 41,2 & A1 \\
\hline Psicologia em Estudo & 16 & 5,4 & 137 & 46,6 & A2 \\
\hline Estudos de Psicologia de Natal & 14 & 4,8 & 151 & 51,4 & A2 \\
\hline Acta Paulista de Enfermagem & 12 & 4,1 & 163 & 55,5 & $\mathrm{~B} 1$ \\
\hline Psico-USF & 12 & 4,1 & 175 & 59,6 & A2 \\
\hline Paidéia & 9 & 3,1 & 184 & 62,7 & A1 \\
\hline Revista Brasileira de Enfermagem & 9 & 3,1 & 193 & 65,8 & $\mathrm{~B} 2$ \\
\hline Revista de Psiquiatria Clínica & 9 & 3,1 & 202 & 68,9 & B3 \\
\hline Cadernos de Saúde Pública & 7 & 2,4 & 209 & 71,3 & A2 \\
\hline Ciência e Saúde Coletiva & 7 & 2,4 & 216 & 73,7 & $\mathrm{~A} 2$ \\
\hline Revista Brasileira de Psiquiatria & 7 & 2,4 & 223 & 76,1 & A2 \\
\hline Revista de Saúde Pública & 6 & 2,0 & 229 & 78,1 & A2 \\
\hline Revista de Psiquiatria do Rio Grande do Sul/Trends in Psychiatry and Psychotherapy & 5 & 1,7 & 234 & 79,8 & B3 \\
\hline Saúde e Sociedade & 5 & 1,7 & 239 & 81,5 & $\mathrm{~B} 2$ \\
\hline Physis: Revista de Saúde Coletiva & 4 & 1,4 & 243 & 82,9 & $\mathrm{~B} 1$ \\
\hline Revista Gaúcha de Enfermagem & 4 & 1,4 & 247 & 84,3 & $\mathrm{~B} 1$ \\
\hline Texto Contexto Enfermagem & 4 & 1,4 & 251 & 85,7 & $\mathrm{~B} 1$ \\
\hline Arquivos de Neuro-Psiquiatria & 3 & 1,0 & 254 & 86,7 & B1 \\
\hline Educação e Pesquisa & 3 & 1,0 & 257 & 87,7 & B1 \\
\hline Psicologia Escolar e Educacional & 3 & 1,0 & 260 & 88,7 & B1 \\
\hline Psicologia USP & 3 & 1,0 & 263 & 89,7 & $\mathrm{~B} 1$ \\
\hline Revista Brasileira de Educação Especial & 3 & 1,0 & 266 & 90,7 & $\mathrm{~B} 1$ \\
\hline Interface: Comunicação, Saúde, Educação & 2 & 0,7 & 268 & 91,4 & $\mathrm{~B} 2$ \\
\hline Psicologia: Ciência e Profissão & 2 & 0,7 & 270 & 92,1 & A2 \\
\hline Revista Brasileira de Educação Médica & 2 & 0,7 & 272 & 92,8 & $\mathrm{~B} 2$ \\
\hline Revista Brasileira de Medicina do Esporte & 2 & 0,7 & 274 & 93,5 & B1 \\
\hline Revista da Associação Médica Brasileira & 2 & 0,7 & 276 & 94,2 & B2 \\
\hline Anais Brasileiros de Dermatologia & 1 & 0,3 & 277 & 94,5 & B1 \\
\hline Arquivos Brasileiros de Cirurgia Digestiva & 1 & 0,3 & 278 & 94,8 & B4 \\
\hline Cadernos CEDES & 1 & 0,3 & 279 & 95,1 & $\mathrm{~B} 1$ \\
\hline Educação em Revista & 1 & 0,3 & 280 & 95,4 & B5 \\
\hline Escola Anna Nery & 1 & 0,3 & 281 & 95,7 & B3 \\
\hline Fractal Revista de Psicologia & 1 & 0,3 & 282 & 96,0 & $\mathrm{~B} 1$ \\
\hline Jornal Brasileiro de Nefrologia & 1 & 0,3 & 283 & 96,3 & -- \\
\hline Jornal Brasileiro de Pneumologia & 1 & 0,3 & 284 & 96,6 & B2 \\
\hline Jornal de Pediatria & 1 & 0,3 & 285 & 96,9 & $\mathrm{~B} 2$ \\
\hline Psychology \& Neuroscience & 1 & 0,3 & 286 & 97,2 & A2 \\
\hline Revista Brasileira de Cirurgia Cardiovascular & 1 & 0,3 & 287 & 97,5 & -- \\
\hline Revista Brasileira de Educação Física e Esporte & 1 & 0,3 & 288 & 97,8 & B2 \\
\hline Revista Brasileira de Ginecologia e Obstetrícia & 1 & 0,3 & 289 & 98,1 & B3 \\
\hline
\end{tabular}


Tabela 2. Periódicos, em frequência decrescente de publicação de artigos, com respectiva avaliação Qualis/CAPES - área Psicologia (2013) Cont.

\begin{tabular}{|c|c|c|c|c|c|}
\hline Periódico & $\mathrm{F}$ & $\%$ & $\begin{array}{c}\mathrm{F} \\
\text { Acumulada }\end{array}$ & $\begin{array}{c}\% \\
\text { Acumulada }\end{array}$ & $\begin{array}{c}\text { Qualis/ } \\
\text { CAPES }\end{array}$ \\
\hline Revista Brasileira de Hematologia e Hemoterapia & 1 & 0,3 & 290 & 98,4 & -- \\
\hline Revista do Hospital das Clínicas/Clinics & 1 & 0,3 & 291 & 98,7 & B1 \\
\hline Revista Estudos Feministas & 1 & 0,3 & 292 & 99,0 & B1 \\
\hline São Paulo Medical Journal & 1 & 0,3 & 293 & 99,3 & -- \\
\hline Trabalho Educação e Saúde & 1 & 0,3 & 294 & 99,6 & B2 \\
\hline Total & 294 & 100 & 294 & 99,6 & -- \\
\hline
\end{tabular}

Por outro lado, ainda analisando os periódicos divulgadores, os da Psicologia foram responsáveis por produção maior de trabalhos publicados sobre coping: 119 (40,5\%), concentrados em 11 títulos ( $M=10,9$ artigos/ periódico). Isto é, apesar de a lista de periódicos da área de Medicina ser numericamente superior em títulos $(n=16)$, a produção sobre coping ali publicada $(12,9 \%)$ está dispersa em uma média de 2,4 artigos/periódico. Nesse sentido, periódicos da Enfermagem publicaram 31,3\% de artigos $(M=13,1$ artigos/periódico), da Saúde Pública/ Coletiva 9,9\% ( $M=5,8$ artigos/periódico), da Educação 3,7\% ( $M=1,8$ artigos/periódico) e Interdisciplinares $1,7 \%$ ( $M=1,2$ artigos/periódico). A prova de Qui-quadrado de aderência indicou diferença significativa para veículos científicos da Psicologia na disseminação do tema coping na amostra estudada $\left(\chi^{2}=217,32 ; \chi^{2 c}=11,07 ; p<0,05 ;\right.$ n.g.l. $\left.=5\right)$, revelando que os periódicos da área da Psicologia publicaram significativamente mais artigos relacionados ao tema coping que os das outras áreas apreciadas.

\section{Autoria dos Documentos}

Autoria é uma variável merecedora de atenção dos avaliadores da ciência, porque se espera certa equidade de participação de pesquisadores, quer seja pelo gênero, quer seja pela preponderância de trabalhos em equipe (Witter, 2008). A análise do material considerou as categorias de autoria masculina, feminina e mista. Em todos os casos foi possível identificar o sexo da autoria. As exceções, quando existentes, foram esclarecidas por meio dos Currículos Lattes dos autores, disponíveis publicamente por meio da Plataforma Lattes.

Trabalhos de autoria feminina foram verificados em $60,5 \%$ dos casos; em $5,1 \%$, masculina e os de autoria mista responderam por $34,4 \%$. O teste $\chi^{2}$ entre os sexos feminino, masculino e misto resultou em 135,68 , portanto, superior ao $\chi 2 c=5,99(p<0,05, n . g . l .=2)$, o que permite inferir que significantemente mais mulheres produziram artigos sobre coping na amostra estudada, quando comparadas aos homens e aos grupos mistos quanto ao sexo. Esse dado, contudo, deve ser mitigado à luz da preponderância feminina na Psicologia em geral, e não somente em relação ao estudo de coping.

Outro indicador que tem sido considerado forte quanto ao desenvolvimento de uma área científica é o quanto os trabalhos são desenvolvidos em equipes. Autorias múltiplas desfrutam de maior prestígio no meio científico quando comparadas às individuais. Dentre outras razões, isso ocorre porque, subjacente a esse tipo de parceria, é possível constatar a existência de grupos e linhas de pesquisa, a integração e cooperação entre instituições e países diversos, algo progressivamente valorizado nos dias atuais, inclusive por órgãos governamentais e de fomento à pesquisa, como a CAPES.

Nesse sentido, a autoria foi analisada quanto às seguintes modalidades, cujos resultados são fornecidos entre os respectivos parêntesis: simples $(n=20 ; 6,8 \%)$, dupla $(n=131$; $44,6 \%)$, tripla $(n=73 ; 24,8 \%)$, quádrupla $(n=33 ; 11,2 \%)$ e com cinco ou mais autores $(n=37 ; 12,6 \%)$. Para verificar se havia diferença estatística entre a ocorrência de trabalhos de autoria múltipla e única foi feito o teste $\chi 2$, que resultou em 137,07 ( $\chi 2 c=9,49 ; p<0,05$, n.g.l. $=4)$ e permite concluir que existem significativamente mais artigos sobre coping publicados por meio de autorias duplas que por meio de outros tipos de autorias.

Se a esse resultado da autoria em duplas considerar-se o restante das ocorrências de trabalhos em autoria múltipla em conjunto (Frequência Acumulada de 93,2\%), há forte indício de bom desenvolvimento da área de produções sobre coping. Quando se retomam estudos de análise de produção científica desenvolvidos na realidade brasileira, o predomínio de autoria feminina e múltipla em profissões como a de Psicologia e Enfermagem não é incomum (Pinto et al., 2011). Além do mais, como visto anteriormente, periódicos da Psicologia e da Enfermagem concentraram o maior volume de publicações sobre coping.

Ressalta-se, ainda, que a distribuição do número de autores por artigos depende da área do conhecimento considerada. Citando Packer (2011), quando a coleção SciELO é considerada, "perto de $50 \%$ dos artigos publicados em 2009 tem até três autores [...]. nos periódicos das ciências agrárias e ciências da saúde predominam os artigos com quatro ou mais autores..." (p. 51). Contextualizando a Psicologia, a Enfermagem e o próprio tema coping na área da saúde, esses achados podem ser melhor compreendidos.

\section{Tipos de Produções}

No que diz respeito à análise dos tipos de estudos, foram consideradas cinco categorias distintas, inspiradas no estudo de Pinto, Santeiro e Santeiro (2010): 1) estudo empírico: pesquisa envolvendo levantamento e análise de dados com a finalidade de conhecer um fenômeno ou de testar uma hipótese. Usualmente é estruturado com base nos itens: introdução, objetivos, método, resultados, discussões e conclusões e envolve a participação de seres humanos; 2) trabalho teórico sistemático: revisão sistemática da literatura 
concernente a um tema ou tópico específico com o objetivo de delinear o estado da arte, identificar principais autores e/ou pesquisas, mostrar a evolução de conhecimentos sobre um tema específico, apontar falhas e acertos dos diversos trabalhos na área, constituindo o resumo do que é realmente importante sobre o tema focado; 3) trabalho teórico assistemático: voltado para a reflexão de um tema, tópico ou conceito teórico, ou para a descrição de uma técnica psicológica. Exclui trabalhos teóricos com ilustrações clínicas, não se tratando, portanto, de pesquisa sistemática como verificado no item 2 acima; 4) estudo de caso: focado no estudo de um caso individual, grupal ou familiar, seja para reflexão de um tema, tópico ou conceito teórico, ou para a descrição de uma técnica e/ou intervenção psicológica. Inclui trabalhos teóricos com ilustrações/resenhas clínicas que utilizam estratégias de avaliação psicológica para coleta de dados; e 5) relato de experiência: aplicação de novos programas ou formas de intervenção desenvolvidas empiricamente e que são descritas por seus autores e/ou praticantes.

Sendo assim, os tipos de produção observados foram, por ordem decrescente: estudos empíricos $(n=226 ; 76,8 \%)$, estudos teóricos assistemáticos $(n=38 ; 12,9 \%)$, estudos teóricos sistemáticos $(n=14 ; 4,8 \%)$, seguidos por relatos de experiência $(n=9 ; 3,1 \%)$ e estudos de caso $(n=7 ; 2,4 \%)$. O teste $\chi 2$ foi feito para verificação de significância estatística quanto às categorias dos tipos de pesquisa e resultou em 604,71 , portanto, marcadamente superior ao $\chi 2 c=9,49$ $(p<0,05, n . g . l .=4)$. Esse resultado permite inferir que estudos empíricos sobre coping, quando comparados aos outros tipos observados, são predominantes na amostra considerada.

Observando os resultados descritos até aqui, como o sexo e a modalidade de autoria, a significância de produções empíricas, além do rigor editorial de periódicos sediados na base de dados SciELO, é preciso considerar que esse conjunto de fatores confere alto valor científico às produções sobre coping. Como se sabe, a ciência é constituída por atividades de pesquisa, que envolvem processos que vão desde a problematização de um tema até a publicação dos resultados junto à comunidade acadêmica e isso valoriza o papel das pesquisas empíricas observadas. Por outro lado, embora estatisticamente menos relevantes, os demais tipos de estudos têm o poder de respaldar e fomentar atividades de pesquisa, o que parece, neste momento, indicar caminhos profícuos aos interessados em aprofundamentos e ampliações sobre a temática do coping.

Como também é de conhecimento geral, a Psicologia brasileira é historicamente marcada por dicotomização entre pesquisa e prática (Gomes, 2003). Esses resultados podem ser reflexos dessa peculiaridade. Naturalmente, na consideração por esse tipo de raciocínio também deve ser ponderado o fato de a SciELO ser uma biblioteca que indexa periódicos científicos. Estes, para serem bem avaliados do ponto de vista da própria comunidade científica, consideram estudos originais como prioridade editorial (SciELO, 2012).

Os achados descritos até aqui não diferem do que tem sido constatado em produções científicas de psicólogos. Costa, Amorim e Costa (2010) constataram em seu estudo, serem generalizadas as conclusões indicando que o número de produções na Psicologia tem crescido significativamente nos últimos anos e que elas têm consistido principalmente de pesquisas, com autorias predominantemente múltiplas e femininas. Quando ponderado o papel da Psicologia na divulgação de estudos sobre coping, esse argumento se consolida.

\section{Coping: Ainda uma Nova Terminologia?}

As observações feitas sobre a especificidade ou a generalidade das produções quanto aos modelos teóricos de coping também foram sistematizadas, a partir do momento em que se adentrou na análise do material e se observou que o uso do termo coping nem sempre era específico. Sendo assim, foi analisado se os artigos especificavam a teoria de coping investigada/explorada, conforme propostas de autores como Lazarus e Folkman (1984) e Moos (2002), ou se coping referia-se à "enfrentamento" a partir de uma linguagem empregada cotidianamente (substantivo masculino que designa ato ou efeito de enfrentar), como, por exemplo, enfrentamento de diferentes situações de vida, enfrentamento de problemas com políticas públicas, enfrentamento do envelhecimento.

Nesse sentido, 120 (40,8\%) artigos buscados eram referentes à ideia genérica de enfrentamento, enquanto grande parte deles $(n=174 ; 59,2 \%)$ fazia referência, de fato, aos modelos teóricos de coping, apresentando especificidade teórica e de critérios de análise do escopo teórico de coping. A prova de Qui-quadrado de aderência indicou diferença significativa para estudos específicos sobre coping, resultando em 9,91 $(\chi 2 c=3,84 ; p<0,05$, n.g.l. $=1)$. Esse resultado destacou que o modelo de Lazarus e Folkman tem forte influência nos estudos sobre o tema, entre autores que buscaram periódicos indexados à SciELO para publicar seus estudos.

\section{Temáticas, Locais e Segmentos Populacionais}

Os 294 estudos sobre coping foram organizados em 13 eixos temáticos, os quais foram estabelecidos por meio de informações disponíveis nos títulos, resumos e palavras-chave dos artigos: 1) competências psicológicas; 2) competências sociais; 3) cuidado à saúde das pessoas; 4) metodologia em Psicologia; 5) questões educacionais; 6) questões sobre desenvolvimento humano; 7) questões sobre o mundo do trabalho; 8) questões socioculturais; 9) transtornos afetivos; 10) transtornos de ansiedade; 11) transtornos predominantemente orgânicos; 12) transtornos relacionados ao abuso de substâncias; 13) outras questões. Aqueles enfocando transtornos predominantemente orgânicos $(17,8 \%)$ foram o eixo temático mais frequente; nesses casos, câncer e a HIV/aids foram dois dos subtemas mais pesquisados.

Coping em questões sobre o desenvolvimento humano foi o segundo eixo temático mais frequentemente enfocado $(17,3 \%)$, sendo os subtemas infância/adolescência e família os mais frequentes. Estudos que focalizam o coping em questões socioculturais foram o terceiro eixo temático mais frequente $(15,6 \%)$, sendo que, nesses casos, os subtemas promoção de 
saúde/qualidade de vida e religião/espiritualidade foram mais frequentemente discutidos.

Sobre os locais onde os estudos foram realizados, eles foram agrupados em sete grandes conjuntos, definidos por meio das informações constantes da metodologia das produções: 1) instituições hospitalares; 2) serviços de saúde pública e mental; 3) familiares/residenciais; 4) organizacionais/empresariais; 5) instituições escolares; 6) outros locais (ruas e praças, abrigos, delegacias etc.; e 7) locais não especificados (ou porque eram estudos teóricos, ou porque o local não foi informado no texto). Nessa análise, estudos realizados em instituições hospitalares foram os mais frequentes $(27,6 \%)$, sendo os hospitais universitários os que receberam destaque, seguidos por serviços de saúde pública e mental $(23,1 \%)$ e por instituições escolares $(16,3 \%)$.

Finalmente, os segmentos populacionais foram organizados em 10 grandes grupos, o que também se deu por meio da análise de informações metodológicas das produções, contudo, mesmo em estudos teóricos essa informação pôde ser considerada: 1) estudantes; 2) famílias de adoecidos/cuidadores/acompanhantes; 3) homens; 4) idosos; 5) mulheres; 6) profissionais da saúde; 7) usuários de serviços de saúde; 8) outros profissionais; 9) outros segmentos (bebês de baixo peso, vítimas de queimaduras, comunidades etc.); e 10) segmento não especificado (estudos teóricos). Usuários de serviços de saúde foram os segmentos mais estudados $(18,2 \%)$. Na sequência, os mais frequentes foram profissionais da saúde $(17,1 \%)$ e outros profissionais $(13,1 \%)$, dentre os quais professores se sobressaíram.

\section{Considerações Finais e Sugestões}

Considerando a SciELO como base de dados representativa de amostra de produções sobre coping, o rigor editorial para que um periódico seja admitido e se mantenha ali cadastrado, e a visibilidade da produção que divulga, este estudo traçou o perfil geral da produção e pode contribuir para que pesquisadores desfrutem de um olhar sistematizado sobre o que vem sendo produzido sobre coping.

Como o estudo foi desenhado para se ocupar de produções divulgadas em periódicos indexados à base de dados SciELO, há que se atentar para o fato de que esses mesmos periódicos também são indexados a outras bases de dados, muitas delas estrangeiras. Assim, considerando o periódico de Psicologia que mais divulgou estudos sobre coping (Psicologia: Reflexão e Crítica), ele é indexado em outras 22 fontes. Esse tipo de observação fortalece a representatividade da amostra estudada, no sentido de que ela abarca experiências que ultrapassam a realidade brasileira.

Em retomada às problematizações que conduziram às investigações e considerando-se os parâmetros metodológicos descritos, pode-se afirmar que a quantidade da produção sobre coping tem se mantido, no geral, em movimento crescente nos 20 anos ora contemplados. É prudente ressaltar que os resultados observados nos anos iniciais de 2010 são parciais. Para serem melhor compreendidos, há que aguardar mais algum tempo para se verificar se há efetivamente um aumento de produção na área, denotando solidificação de linhas de pesquisa sobre essa temática entre aqueles que buscam publicar seus trabalhos em veículos indexados à SciELO. Essa cautela é necessária, já que existem hiatos entre períodos de conclusão de relatórios de pesquisa e a publicação efetiva de seus resultados na forma de artigos. Os estudos têm sido divulgados majoritariamente em veículos de responsabilidade editorial da Psicologia, seguidos pelos da Enfermagem, sendo que, no conjunto, periódicos da Psicologia publicaram quase metade da produção analisada $(40,5 \%)$.

Novas investigações permitiriam explorar se o predomínio de autoria em dupla se relaciona à publicação de artigos decorrentes de dissertações e teses, já que, no geral, os programas de pós-graduação têm progressivamente feito a opção por substituir textos em formato monográfico por artigos, como critério de aprovação de seus estudantes. Essa característica da produção coletiva pode, além disso, ser contextualizada como estratégias que os autores têm buscado para lidar com pressões institucionais e profissionais. De modo igualmente marcante, esses estudos sobre coping têm se consolidado por meio de pesquisas empíricas.

Este relato foi referente a uma análise sistemática de parcela de artigos científicos publicados sobre coping, com finalidade de mapeamento geral da produção divulgada na base de dados SciELO. Outros aprofundamentos podem ser feitos, especialmente dispensando-se atenção aos artigos específicos sobre coping aqui detectados. Verificar a afiliação institucional dos seus autores, a existência de redes de colaboração sobre coping e analisar mais detidamente as pesquisas empíricas desenvolvidas, seriam algumas possibilidades.

\section{Referências}

Aros, M. S., \& Yoshida, E. M. P. (2009). Estudos de depressão: Instrumentos de avaliação e gênero. Boletim de Psicologia, LIX(130), 61-76.

Barroso, S. M. (2010). Avaliação psicológica: análise das publicações disponíveis na SciELO e BVS-Psi. Fractal: Revista de Psicologia, 22(1), 141-154.

Bordens, K. S., \& Abbott, B. B. (1999). Research design and methods: A process approach (4a ed.). Mountain View (CA): Mayfield Publishing Company.

Coordenação de Aperfeiçoamento de Pessoal de Nível Superior [CAPES] (2013). Qualis periódicos. Brasília: CAPES. Retrieved from http://capes.gov.br/avaliacao/qualis .

Costa, A. L. F., Amorim, K. M. O., \& Costa, J. P. (2010). Profissão de psicólogo no Brasil: análise da produção científica em artigos. In O. H. Yamamoto \& A. L. F. Costa (Eds.), Escritos sobre a profissão de psicólogo no Brasil (pp. 31-58). Natal: EDUFRN.

Custódio, Z. A. O., Crepaldi, M. A, \& Cruz, R. M. (2012). Desenvolvimento de crianças nascidas pré-termo avaliado pelo teste de Denver-II: revisão da produção científica brasileira. Psicologia: Reflexão e Crítica, 25(2), 400-406.

Domingos, N. A. M. (1999). Perspectivas da produção científica da pós-graduação em psicologia da PUC-Campinas. In G. P. Witter (Ed.), Produção cientifica em Psicologia e Educação (pp. 79-102). Campinas, SP: Alínea.

Folkman, S., \& Lazarus, R. S. (1980). An analysis of coping in a middle-aged community sample. Journal of Health and Social Behavior, 21, 219-239. 
Gomes, W. B. (2003). Pesquisa e prática em psicologia no Brasil. In O. H. Yamamoto \& V. V. Gouveia (Eds.), Construindo a psicologia brasileira: desafios da ciência e da prática psicológica (pp. 23-59). São Paulo: Casa do Psicólogo.

Granja, E. C. (1995). Produção científica: Dissertações e teses do Instituto de Psicologia da USP (1980/1989). (Unpublished doctoral dissertation). Universidade de São Paulo, São Paulo, SP.

Herculano, R. D., \& Norberto, A. M. Q. (2012). Análise da produtividade científica dos docentes da Universidade Estadual Paulista, Campus de Marília/SP. Perspectivas em Ciência da Informação, 17(2), 57-70.

Joly, M. C. R. A., Berberian, A. A., Andrade, R. G., \& Teixeira, T. C. (2010). Análise de teses e dissertações em avaliação psicológica disponíveis na BVS-PSI Brasil. Psicologia: Ciência Profissão, 30(1), 174-187.

Lazarus, R. S., \& Folkman, S. (1984). Stress, appraisal, and coping. New York: Springer.

Massi, L., \& Queiroz, S. L. (2012). Investigando processos de autoria na produção do relatório de Iniciação Científica de um graduando em Química. Ciência \& Educação (Bauru), 18(2), 271-290.

Moos, R. H. (1993). Coping response inventory youth form Professional manual. Odessa: PAR Psychological Assessment Resources.

Moos, R. H. (2002). The mystery of human context and coping: An unraveling of clues. American Journal of Community Psychology, 30(1), 67-88.

Moos, R. H. (2003). Social context: Transcending their power and their fragility. American Journal of Community Psychology, 31(2), 1-13.

Packer, A. L. (2009). The SciELO open access: A gold way from the South. Canadian Journal of Higher Education, 39(3), 111-126.

Packer, A. L. (2011). Os periódicos brasileiros e a comunicação da pesquisa nacional. Revista USP, 89, 26-61.
Packer, A. L., Biojone, M. R., Antonio, I. T. R. M., García, A. P., Silva, A. C., Murasaki, R. Delbucio, H. (1998). SciELO: uma metodologia para publicação eletrônica. Ciência da Informação, 27(2), 109-121.

Pinto, L. P., de Lima, T. H., \& de Lima, R. C. (2011). Estudo da produção científica da Revista Psico-USF de 2007 a 2011. Psico-USF, 16(3), 383-390.

Pinto, F. P., Santeiro, T. V., \& Santeiro, F. R. M. (2010). Produção científica sobre psicoterapias na base de dados PePsic (1998/2007). Fractal: Revista de Psicologia, 22(2), 411-430.

Poblacion, D. A., Witter, G. P., \& Silva, J. F. M. (Eds.) (2006). Comunicação \& produção científica: Contexto, indicadores e avaliação. São Paulo: Angellara.

Sabadini, A. Z. P., Sampaio, M. I. C., \& Koller, S. H. (Eds.) (2009). Publicar em Psicologia: Um enfoque para a revista cientifica. São Paulo: Associação Brasileira de Editores Científicos de Psicologia; Instituto de Psicologia da Universidade de São Paulo.

Sampaio, M. I. C., \& Serradas, A. (2009). O movimento de acesso aberto, os repositórios e as revistas científicas. In A. Z. P. Sabadini, M. I. C. Sampaio \& S. H. Koller (Eds.), Publicar em Psicologia: Um enfoque para a revista cientifica (pp. 7586). São Paulo: Associação Brasileira de Editores Científicos de Psicologia; Instituto de Psicologia da Universidade de São Paulo.

Scientific Electronic Library Online. (2012). Sobre o SciELO: Modelo SciELO. São Paulo: FAPESP; BIREME. Retrieved from http://www.scielo.org/php/level.php?lang=pt\&compon ent $=56$ \&item $=1$

Siegel, S. (1975). Estatística não-paramétrica para as ciências do comportamento. Trad. Alfredo Alves de Farias. São Paulo: McGraw-Hill. (Trabalho original publicado em 1956)

Witter, G. P. (2008). Psicologia da saúde e produção científica. Estudos de Psicologia (Campinas), 25(4), 577-584.

Yoshida, E. M. P., Santeiro, T. V., Santeiro, F. R. M., \& Rocha, G. M. A. (2005). Psicoterapias breves psicodinâmicas: características da produção científica nacional e estrangeira (1980/2003). Psico-USF, 10(1), 51-59. 